\title{
Improving PMTCT Coverage and Access in Communities with Unmet Needs in Jos, Nigeria by Adopting Task Shifting and Task Sharing Strategies
}

\author{
Tinuade Abimbola Oyebode ${ }^{1,2, ~ *}$, Zuwaira Hassan ${ }^{1,3}$, Tolulope Afolaranmi ${ }^{1,3}$, Muazu Auwal ${ }^{4}$, \\ Mohammed Shehu ${ }^{5}$, Ngwoke Kelechi ${ }^{6}$, Agbaji Oche ${ }^{1,7}$, Solomon Sagay ${ }^{1,2}$, Jerry Gwamna ${ }^{8}$, \\ Prosper Okonkwo ${ }^{6}$, Phyllis Kanki ${ }^{9}$
}

\author{
${ }^{1}$ Faculty of Medical Sciences, University of Jos, Jos, Nigeria \\ ${ }^{2}$ Department of Obstetrics and Gynaecology, Jos University Teaching Hospital, Jos, Nigeria \\ ${ }^{3}$ Department of Community Medicine, Jos University Teaching Hospital, Jos, Nigeria \\ ${ }^{4}$ Department of Chemical Pathology, Jos University Teaching Hospital, Jos, Nigeria \\ ${ }^{5}$ Department of Strategic Information (Data), Jos University Teaching Hospital, Jos, Nigeria \\ ${ }^{6}$ AIDS Prevention Initiative in Nigeria (APIN), Abuja, Nigeria \\ ${ }^{7}$ Department of Internal Medicine, Jos University Teaching Hospital, Jos, Nigeria \\ ${ }^{8}$ Center for Disease Control and Prevention (CDC), Abuja, Nigeria \\ ${ }^{9}$ Infectious Diseases \& Immunology, Harvard School of Public Health, Boston, MA, USA
}

\section{Email address:}

tinuadeoyebode@yahoo.com (T. A. Oyebode), zuwairahassan@yahoo.com (Z. Hassan), toluene42002@gmail.com (T. Afolaranmi), muazuma2@yahoo.com (M. Auwal), nibrass2@yahoo.com (M. Shehu),kngwoke@apin.org.ng (N. Kelechi), oagbaji@yahoo.com (A. Oche), atsagay@yahoo.com (S. Sagay), GwamnaJ@ng.cdc.gov (J. Gwamna), pokonkwo@apin.org.ng (P. Okonkwo),pkanki@hsph.harvard.edu (P. Kanki)

${ }^{*}$ Corresponding author

\section{To cite this article:}

Tinuade Abimbola Oyebode, Zuwaira Hassan, Tolulope Afolaranmi, Muazu Auwal, Mohammed Shehu, Ngwoke Kelechi, Agbaji Oche, Solomon Sagay, Jerry Gwamna, Prosper Okonkwo, Phyllis Kanki. Improving PMTCT Coverage and Access in Communities with Unmet Needs in Jos, Nigeria by Adopting Task Shifting and Task Sharing Strategies. European Journal of Preventive Medicine. Vol. 9, No. 3, 2021, pp. 83-93. doi: 10.11648/j.ejpm.20210903.13

Received: April 28, 2021; Accepted: May 14, 2021; Published: May 26, 2021

\begin{abstract}
Towards achieving an AIDS-free generation, UNAIDS set the 90-90-90 target aiming at 90\% of HIV positive persons knowing their status, $90 \%$ of positives receive sustained antiretroviral drugs and $90 \%$ of those receiving ARVs attain virologic suppression by 2020 . The attainment are dependent on continual access, quality care and treatment retention, so efforts must address context specific barriers to accessing services. The ethnoreligious conflicts in Jos created barriers to accessing HIV/PMTCT services, even when treatment sites existed around the metropolis. Fifteen communities lacked comprehensive HIV services and residents could not access treatment facilities because of security challenges. A specialized strategy using community oriented resource persons (CORPs) and task shifting task sharing (TSTS) principles conceptualized by stakeholders was utilized to bridge personnel gaps and scale-up PMTCT. The HIV Lead Implementing Partner supported a faith based community organization to identify and scale-up PMTCT into 28 hospitals in 15 communities. Training and task devolution to Community Health workers (CHWs), expert patients and Traditional Birth Attendants (TBAs) was utilized. The facilities were networked for service delivery, referrals, supervision and commodity logistics. HIV testing was provided to pregnant women during ANC, labour and postnatal, and their children and spouses. All 28 facilities offered HCT and provided ARVs to those testing positive in labour, women testing positive during ANC were managed/referred to 8 PMTCT sites for evaluation and ARV commencement according to Nigerian HIV Guidelines. Infants received Nevirapine, early infant diagnosis and Cotrimoxazole. HIV positive children and non-pregnant adults were referred to three ART sites for evaluation and treatment. The twenty-eight facilities were activated for HCT/PMTCT/ART using MNCH structures and CHEWs, TBAs and PLHIV expert patients provided care, support and tracking. After the six-month pilot, of 3,293 women receiving ANC, 3,094
\end{abstract}


(93.9\%) accepted HCT and received same-day results. Thirty-four tested positive, but 15 previously knew their status and on ARVs, but had challenges accessing care, while 17 of 19 newly diagnosed women commenced ARVs while 2 defaulters are being tracked. Five HIV exposed babies delivered received Nevirapine and cotrimoxazole, four were tested HIV-negative. Also 7193 adults and 23 children received HCT and results, 69 positive adults and 2 positive children enrolled care, among who 33 adults and 2 children commenced ARVs. PMTCT diagnostics must identify specific barriers communities experience and implement multipronged context specific scale-up efforts to improve access/uptake to eliminate Paediatric HIV infections. CORPs and TSTS strategies are critical to improve service-delivery and retention in care.

Keywords: Task Shifting Task Sharing, Devolve, Community Resources, PMTCT, Scale-up

\section{Background}

UNAIDS set an ambitious target code named 90-90-90 which aims to ensure that $90 \%$ of all people with HIV know their status, $90 \%$ of those diagnosed will receive sustained ART and $90 \%$ of those on ART attain virologic suppression [1] among efforts towards achieving an AIDS free generation. Antiretroviral prophylaxis has prevented over 350,000 children from acquiring HIV infection globally each year between 1995 and 2010 , and $86 \%$ of children who avoided infection, live in sub-Saharan Africa, the region with the highest HIV prevalence among women of reproductive age [2]. Nigeria still lags behind on attaining the UNAIDS targets because of areas without access to HIV/PMTCT, and challenges like conflict were responsible for non-attainment of the MDG targets [3].

Plateau is the twelfth largest state of Nigeria, and it is geographically unique in because Jos Plateau is totally in the central and northern part of Nigeria. Its capital is Jos and has been divided into Jos North, Jos South and Jos East [4]. The focus areas are majorly in Jos North, with a population of 437,217 persons according to the 2006 Census [5]. The state has been affected by series of ethnoreligious crises since 2001, which has changed living condition of the populace. The crises created trends of distrust that led to partitioning of the populace, with people refraining from assessing parts of Jos where the major HIV comprehensive facilities are situated, creating a barrier to accessing PMTCT/HIV services for these communities. The crises had created a synergy of displacement of populations, large numbers of out-of-school girls, loss of livelihoods, poverty, increased transactional sex and sexual assault and deployment of large numbers of armed personnel increased the risk for HIV transmission. The observations led to stakeholders' deliberations, facility mappings, HIV/PMTCT diagnostics and identification of communities lacking PMTCT/HIV treatment centers, who found it difficult to access other parts of Jos metropolis.

Humanitarian crisis include predictable or unpredictable, sudden or slow onset natural disasters, armed conflict or civil disturbance. These may result in complex emergencies and can be recurrent in chronically fragile and post-conflict settings. Contexts may differ but they all pose critical threats to health, safety and security of many people, often with people displacement that affects healthcare delivery. Potential negative impact on PMTCT programs include access disruption of access to functional PMTCT and broader SRH services, treatment interruption which increased risk of antiretroviral resistance, poor access to ART for PMTCT which may contribute to increases in new paediatric HIV infections [6].

In the background of challenges including security, Nigeria also faces one of the worst human resources for health (HRH) crises worldwide, which in the backdrop of insecurity, constitutes a double tragedy to be considered ahead of planning scale up of HIV/PMTCT. The HCW to population density is below WHO recommendations of 23 per 10,000 population, and $\mathrm{HCW}$ are poorly distributed, in favour of urban, southern, tertiary hospitals and curative care [7]. Nigeria Health workers profile, as of December 2012 [8] documented that 20,284 Nigerian medical doctors were in good standing, and their densities per 100,000 populations ranged from 50.5 (FCT) to 1.9 in Yobe State, the median state having 8.9 medical doctors per 100,000 (Sokoto). The profile also revealed densities of nurses and midwives taken together per 100,000 population, ranges from 5.9 (Zamfara) to 96.5 (Imo), with 24.7 per 100,000 (Niger) as median.

The National Task shifting task sharing policy [9] documents that Government's decisions to scale-up HIV services and increase ART access was faced with background HRH shortages and traditional Human Resources for Health role specifications were major challenges to expansion. The few ART sites which were centralized and majorly in tertiary hospitals were overstretched from high patient numbers, long waiting times and high $\mathrm{HRH}$ attrition rates. High transportation costs for patients living far, high patient-todoctor ratios, high default rates among PLHIVs on ART and diminishing patient satisfaction raised concerns about quality of care provided by major treatment centers and created a need to decentralize and devolve ART services to expand care and patient access. In 2009, the FMOH recommended a decentralization strategy including shifting HIV management tasks from physicians to non-physician providers, from nurses to CHEWs and subsequently to trained peer educators, patients and communities, thus rapidly increased number of ART/PMTCT sites in Nigeria. ART provision at PHCs and community brought closer to patients' homes and reduce travel time and cost to access care.

In response to these issues, the national Strategic Plan for 2010-2015 listed HRH as one of the eight priority areas, proposing task-shifting as one of the key activities to increase access to healthcare service [10]. In HIV context, TSTS allows specialized health workers to focus on advanced 
clinical conditions, while non-physician HCW care for stable patients and engages the community to care for its own. The National TSTS policy recommendation 20 indicates categories of Community Health Workers including Community Health Officers (CHOs), community health extension workers (CHEWs) and Junior CHEWs can participate in HIV/PMTCT service delivery. Nigeria has approximately 20 CHEWs per population of 10,000, representing a largely untapped resource for maternal and child health $(\mathrm{MNCH})$ care, and recommendation 21 of the TSTS policy indicates that experienced PLHIVs can provide self-care and support services including self-care to other PLHIVs in the facility and community [9].

Regarding HIV/AIDS services, the TSTS policy recommends that CHEWs and PLHIVs can assess client's HIV knowledge, discuss combination therapy, ART goals and importance of medication adherence, identify need for other services and refer appropriately. They will discuss client's medication experience, identify challenges with medication, develop strategies to manage them and achieve adherence. CHEWs can assess patients for adverse drug effects and refer to physicians for further management. Regarding clinical consultation, the TSTT policy allows CHEWs and PLHIVs to initiate treatment for acute symptoms and treatable conditions, follow-up monitoring and manage side-effects for clients started on ART, determine special needs and refer for specialized services using referral forms. CHEWs can clerk and conduct physical examination to assess health status and fill items 8-37 of the initial clinical encounter (ICE) form.

Medical records recommendations allows patient registration to be devolved from officers to assistants, CHEWs and PLWHA, who can register new patient in preART register, assign correct unique number, fill biodata part of PMM forms, issue patient appointment card, schedule client for clinician appointment, fill ART clinic attendance register, compile missed appointments and file folders appropriately. Some ARV dispending services can be devolved from Pharmacists to Pharmacist Technicians, Pharmacist Assistants and PLHIVs. Technicians can document quantities of items dispensed, prepare prescription labels and containers according to requirements, dispense drugs according to prescription information and verify prescription for authenticity, completeness and identify prescription anomalies. PLHIVs are allowed to pre-package prescription items, transfer information accurately from prescriptions and generate monthly summary forms.

Regarding ART laboratory services, TSTS policy recommends some laboratory functions to be devolved from Laboratory Scientist to Laboratory Technician, Laboratory Assistant and PLHIVs. The PLHIVs can anchor activities including preparing items for sample collection, labelling sample containers for sample collection and preparing of patients, collecting patient samples into appropriate containers and registration in specimen receipt register, samples separation for analysis in the ART laboratory, preparation of month-end laboratory data and fill requisition forms for RTKs and reagents. However, appropriate trainings should precede devolution of tasks, and continual on the job supportive supervision should be provided by staff of senior cadre.

\section{Introduction}

A Prevention of Mother to Child Transmission of HIV (PMTCT/HIV) intervention pilot project targeting fifteen communities of Jos, Plateau State capital lacking access to parts of town because of security risks arising from ethnoreligious conflict and the subsequent partitioning. There were seven major Comprehensive HIV treatment facilities, but located in majorly Christian dominated parts of Jos, and three of them were Church based hospitals. These densely populated communities that lacked comprehensive HIV treatment centers included Ali Kazaure, Angwan Rogo, Angwan Rimi, Bauchi Road, Bukuru, Congo Russia, Corner Shagari, Dogon Dutse, Fillin Ball, Gangare, Naraguta Village, Masalacin Jumaa, Masalacin Idi, Nasarawa Gwom, Rikkos and Yan Shanu. This was happening in the backdrop of Nigeria's HIV unmet needs and these gaps required scaling-up HIV/PMTCT services to improve coverage, access and uptake towards PMTCT and elimination Paediatric HIV infections. The peculiar challenges arising from inability of persons of different ethnoreligious dispensations to freely access these communities constituted a special scenario requiring contextualized scale-up strategies.

The Lead HIV Implementing partner (Lead IP), AIDS Prevention Initiative in Nigeria (APIN) and the Plateau State Technical HIV Team consisting all HIV stakeholders, convened a stakeholders meeting to address this challenge and identify strategies to close the gaps. A team of Competent HIV care providers of same faith and ethnicity were engaged to pioneer an intervention through a faith based community organization to scale up HIV/PMTCT using $\mathrm{MNCH}$ platform. They identified, upgraded, activated and trained staff of twenty eight private community facilities to implement programs of HIV testing, Prevention of Mother to Child transmission and Antiretroviral treatment. They engaged, trained and worked with other community oriented resource persons (CORPs)- Persons living with HIV (PLHIVs), Traditional Birth attendants (TBAs) and Muslim women using task shifting and task sharing principles to address the shortage of human resources for health (HRH) in the communities.

\subsection{Program Coverage and Target Population}

The program covered Jos North majorly, which had an estimated population of 794,000 persons in 2014 with an annual growth rate ranging 1.9 and $2.4 \%[11]$. A total of twenty-eight facilities were identified for the project, twentyfive located in Jos North and three in Bukuru (Jos South). The primary target consist pregnant women and their unborn babies, mother/baby pairs in the postnatal population while secondary targets consist household members of infected persons including spouses, children and other family members and other affected community members. 


\subsection{Project Aims and Objectives}

The overall objective of this project is to improve access and contribute towards the improvement of maternal, child and new-born health and survival through increasing the uptake of prevention of mother to child HIV transmission services and providing care and support to infected parents and children using community resource persons and family centered strategies, in communities that have been affected by ethnoreligious crises.

The strategic objectives of the MUHIN project are as follows

1) To promote innovative community based primary prevention of HIV through scale up HIV/PMTCT interventions to pregnant women and their families (Prong 1).

2) To prevent unwanted pregnancies among HIV positive women by promoting use of modern contraceptives (Prong 2).

3) To prevent HIV transmission from pregnant or lactating women living with HIV to their babies by early identification and provision of PMTCT interventions (Prong 3).

4) To mobilize communities and identify female PLHIVS and their families to receive HIV Care, Treatment and Support (Prong 4).

5) To set up specific community systems utilizing acceptable, accessible and affordable services to close HIV/PMTCT gaps in communities affected by ethnoreligious crises.

6) To enhance advocacy, engagement, capacity building, systems development and behaviour change communication for community PMTCT/HIV treatment services.

\section{Methods}

The Project implementation strategy utilized the program cycle approach of assessment, planning, implementation, and monitoring and evaluation. The team and strategy was set up at the beginning of the project.

\subsection{Assessment}

This entailed mapping existing HIV facilities, statewide facility assessment data review and identifying communities lacking HIV/PMTCT services.

A review of unpublished results of rapid statewide facility assessment conducted as part of Plateau PMTCT diagnostics in 2013, revealed that many health facilities were destroyed, healthcare workers ( $\mathrm{HCW})$ were displaced and killed in some communities, while ARV/commodity logistics were disrupted in several parts of the state. Mapping revealed seven PEPFAR supported Comprehensive HIV treatment sites within Jos Metropolis were Jos University Teaching Hospital (JUTH), Plateau State Specialist Hospital (PSSH), Our Lady of Apostles Hospital (OLA), Bingham University Teaching Hospital (BhUTH), Faith Alive Foundation (FAF), SOLAT hospital and Comprehensive health Centre, Dadin Kowa, Jos. None of them was located within the identified disadvantaged communities.

\subsection{Planning}

This included site selection, linkage Model, engaging Staff and volunteers, capacity building, facility upgrade, logistics planning, and providing Monitoring and Evaluation tools.

\subsubsection{Facility Assessment, Selection and Linkages}

A list of fifty-seven appropriate community $\mathrm{MNCH}$ facilities was generated. They were assessed and twenty eight facilities were selected for HIV/PMTCT scale-up, using existing $\mathrm{MNCH}$ service points as entry. The human and institutional capacity, facility location, client flow, new monthly ANC attendees ( $>20$ monthly), facility headship and $\mathrm{MNCH}$ structures were among selection criteria. Two female PLHIVs would work with facility leads to anchor referrals and linkages among sites. Samples would be pooled and taken to the Laboratory for Chemistry, $\mathrm{CD}_{4}, \mathrm{CD}_{4} \%$, viral load and EID. Mentorship and on the job support will be provided by the core MUHIN Team (monthly), with support from Lead IP staff while Laboratory investigations will be taken to Our Lady of Apostles hospital laboratory.

\subsubsection{Implementing Team, Platform and Partnerships}

A team of six health workers who doubled as community leaders, youth religious leader and female PLHIV, of same ethnicity and religion, and residing or relating with affected communities were identified. They consisted a female public health/HIV physician and member of Federation of Muslim Women Association of Nigeria (FOMWAN), a Medical doctor and owner of a community private clinic, a trained HIV Laboratory scientist, a Data/Monitoring and evaluation staff, all of whom had extensive experience in HIV/AIDS program management. The sixth member was a female PLHIV, expert patient, HIV support group member and community women leader who had successfully completed PMTCT program. They set up a faith based community organization, Muslim Health Initiative in Nigeria (MUHIN), registered it with State Ministry of Health (SMOH), Ministry of Women Affairs and Corporate Affairs Commission (CAC). They would work with stakeholders to champion the community entry and project implementation. Partnerships were established between MUHIN, community gatekeepers and HIV stakeholders including Ministry of Health, State AIDS/STI control Program (SASCP), State AIDS control agency (SACA), Primary Healthcare development board, HIV researchers, the Lead HIV IP (AIDS Prevention Initiative in Nigeria), Civil society organizations like CISHAN (Civil Societies for HIV and AIDS in Nigeria), Religious groups including FOMWAN, TBAs, and PLHIVs support groups including ASHWAN (Association of Women living with HIV/AIDS in Nigeria). The Lead IP engaged MUHIN to anchor HIV/PMTCT interventions within target communities. Memorandum of organization was signed, work plans for training, facility set-up and targets were 
generated for MUHIN. Programme areas for implementation were HCT and prevention, PMTCT, Adult ART, Care and support, Paediatric Treatment, TB/HIV co-infection, Laboratory services, Strategic information and financial management.

\subsubsection{Resource Persons and Facility Upgrades}

The MUHIN team engaged facility heads, provided facility upgrades, systems strengthening and developed community systems to support and integrate HIV/MNCH services. They provided personal protective equipment (PPE), universal precaution and Emergency Obstetric and newborn care (EmONC) supplies. The facilities received technical assistance, capacity development to become seventeen HCT sites, eight PMTCT sites, and three comprehensive ART sites. They created linkages using the Hub and spoke model, so that HCT sites fed into PMTCT sites which fed into comprehensive ART sites for patient referrals and commodities logistics. Considering Nigeria's HRH challenges especially in conflict environments, the Nigerian TSTS policy principles were utilized, and CHEWS, TBAs and PLHIV expert patients were involved in facility and community service delivery to improve quality and retention in care.

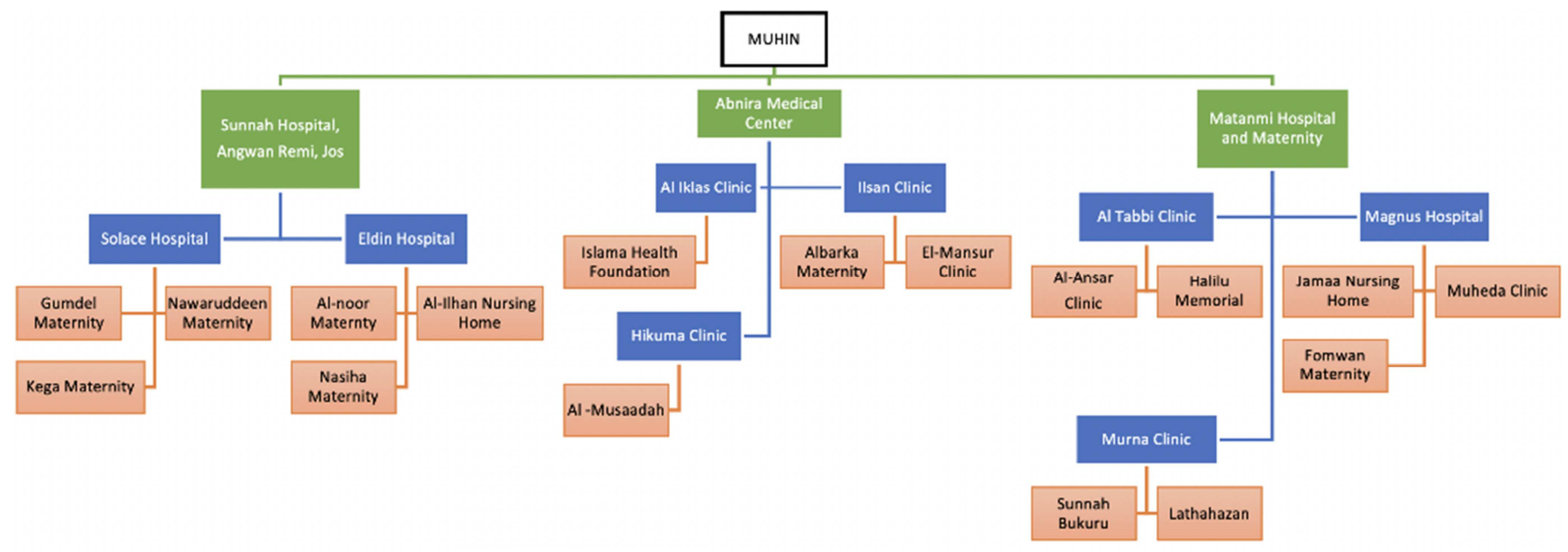

**The ART sites are in Green, PMTCT sites are in Blue and HCT sites are in Red

Figure 1. Hub and Spoke Model of Linking HCT facility to PMTCT facility to ART facility.

\subsection{Implementation}

Commencement of service delivery and $\mathrm{HCT} / \mathrm{PMTCT} / \mathrm{ART}$ and integrated supportive supervision and community programs.

\subsubsection{HCT}

Provider initiated HCT was offered to all women assessing Antenatal care at all 28 project facilities. They received group pre-test counselling and individual posttest counselling. Testing was provided with same day result within antenatal clinics in spaces with audio-visual privacy. The National HCT algorithm was utilized and tests conducted with Determine rapid test Kit (RTK) Strips. Those testing negative received individual posttest counselling, while those testing positive had repeat testing with Unigold rapid test. However, when discordance occurred with Determine and Unigold tests, tie breaker test was performed with stat pack, and confirmed positives offered PMTCT enrolment onsite or by referral within the MUHIN network. HCT was offered to women presenting in labour as unbooked cases, and ARVs provided for PMTCT according to the national guidelines. Opportunity for HCT was offered to children and spouses of female PLHIVs and couple HCT was offered/provided to couples.

\subsubsection{PMTCT}

This was implemented according to the 2010 National PMTCT Guideline [12], which entailed providing ARVs consisting Lamivudine, Zidovudine and Nevirapine to all HIV positive women during ANC, labour and delivery at the eight PMTCT and three ART sites. They had preliminary investigations including chemistry, haematology, $\mathrm{CD}_{4}$ and Viral load, screening for Hepatitis B, $\mathrm{C}$ and sexually transmitted infections (STIs). All samples were pooled to ART Hubs and taken to OLA hospital laboratory and results returned through same route. Women where commenced on haematinics, sulphadoxinepyrimethamine and triple ARVs, but those whose $\mathrm{CD}_{4}>350$ cells $/ \mathrm{mm}^{3}$ discontinued ARVs after pregnancy and complete cessation of breastfeeding according to the PMTCT Guidelines (Option B), while those with $\mathrm{CD}_{4}<350$ cells $/ \mathrm{mm}^{3}$ continued ARVs for life and received cotrimoxazole. Mothers received infant feeding counseling, with emphasis on avoiding mixed feeding and breastfeeding when nipple/breast disease was present. Exclusive breastfeeding or breast milk substitutes were assessed to be affordable, feasible, acceptable, sustainable and safe (AFASS) and supported. All HIV exposed babies received Nevirapine from birth to six weeks, early infant diagnosis (EID) at six weeks, and commenced cotrimoxazole 
thereafter. They would receive HCT by RTK at eighteen months and be discharged from PMTCT, while any child testing positive would be enrolled into paediatric care.

\subsubsection{ART and Care}

Three ART sites provided long term HIV management for adults and children, including lifelong triple ARVs, screening and treatment for opportunistic infections, clinical, immunologic, virologic and other laboratory monitoring of treatment. Adopting the genealogy approach other children of HIV positive women were offered HCT and those tested positive referred to ART sites for evaluation and Paediatrics ART. They had investigations conducted at baseline, then three monthly and subsequently six monthly. They also had screening for Hepatitis B and C, tuberculosis and STIs and provided treatment appropriately. Adherence counseling was provided by pharmacists on site and treatment support provided by experienced PLHIVs.

Facility staff received technical assistance across all program areas from the MUHON leadership, who received support from APIN, the lead IP.

\subsubsection{Community Efforts}

The MUHIN team, facility staff, expert patients and community resource persons formed outreach teams and provided monthly HCT outreaches and referred positive persons to PMTCT or ART treatment sites. The MUHIN team met with various community stakeholders and groups for awareness creation, demand generation and advocacy. A MUHIN support group of identified PLHIVs was set up and started meeting regularly, and expert patients provided adherence counseling, tracking and mother-to-mother (M2M) support.

\subsection{Monitoring and Evaluation}

The $\mathrm{M} / \mathrm{E}$ and data capture training was provided for facility data clerks and each patient is captured with project specific identifiers and National HIV M/E tools at each site. Project facilities received Laboratory and pharmacy commodities and provided Monthly and quarterly progress reports were provided to the lead IP. The MUHIN team doctors provided Quality Improvement/Quality assurance checks with support from the Lead IP QI/QA focal person. They performed random chart checks and performance measurement across clinical, laboratory and other indicators to ensure quality service delivery.

\section{Project Results}

The MUHIN organization was the sub-grantee that engaged and upgraded twenty eight sites, provided equipment and personnel training to provide HCT/PMTCT/ART services, using the hub and spoke approach and provided project reports. The services improved quality and documentation of $\mathrm{MNCH}$ services in addition to bringing HIV/PMTCT services into the target communities.

\subsection{Human Resources for Health (HRH) Capacity Development}

\subsubsection{HCT Training}

This was provided to forty-six nurses, CHWs and PLHIVs, while forty-five received Injection safety training. Twentyfive facility waste handlers received waste management training.

\subsubsection{PMTCT Training}

The Integrated Management of Adolescent and Adult Illness/Integrated Management of pregnancy and childbirth/PMTCT (IMAI/IMPAC/PMTCT) training was conducted for 33 nurses, $\mathrm{CHOs}$ and $\mathrm{CHEWs}$ to build $\mathrm{MNCH}$ and HIV capacity across eight PMTCT and three comprehensive ART sites.

\subsubsection{Adult ART Training}

This was provided to 25 staff including doctors, nurses, pharmacy technicians and CHEWs. Training on Paediatrics Treatment was conducted for six doctors and three nurses from the three ART sites using National Paediatric Training modules. Training on national monitoring and evaluation tools was provided during the HCT, PMTCT and ART to 27 Records clerks and CHEWs.

\subsubsection{Laboratory Training}

Training for sample collection and cataloguing was provided for eight laboratory personnel (consisting a scientist, technicians and CHEWs) from the PMTCT and ART sites.

\subsubsection{Community Resource Persons Training}

They consisted fifty-two PLHIVs, TBAs and FOMWAN members and were attached to the twenty-eight sites and received infection prevention and home based care trainings to enable them provide HCT, tracking, adherence counselling, and community home based care and treatment support. They received monthly stipends for phone credit and transport fare.

\subsubsection{Infrastructure, Equipment and Site Development for MNCH/HIV Services}

MUHIN facilities received furniture for ANC waiting area, thermometers, foetoscopes, stethoscopes, weighing scales and Sphygmomanometers for $\mathrm{MNCH}$ and other hospital patients. They received EmONC materials and Personal protective equipment for maternity areas including veronica buckets, liquid soap, paper towels, cotton wool, methylated spirit, universal precaution materials including aprons, boots, gloves, goggles, mackintosh, injection safety boxes, bin boxes and bags for medical/medical waste, buckets, cleaning utensils, disinfectants and bleach. Labour wards received kidney dishes and tissue forceps, while Job aids, charts, standard operating procedures and National guidelines covering HCT, PMTCT and ART were provided.

\subsubsection{Commodity and ARV Logistics}

Systems were developed for medical supplies using the hub and spoke model where the three ART hospitals were hubs. They received RTKs, ARVs and drugs for 
Opportunistic infections from the MUHIN team and distributed to their respective spoke facilities. Lead IP focal pharmacist and MUHIN team coordinated monthly forecast, distribution and provided monthly reports.

\subsubsection{Monitoring and Evaluation Systems}

Facility records clerks/CHEWs gained competency in client enrolment, providing facility/project identifiers for HCT, PMTCT, HIV Exposed infants and ART, and confidential storage of patients records. Sites received patient folders and National M/E tools for HCT, PMTCT and ART. Facility staff were supported, attained proficiency and utilization commenced.

\subsubsection{Governance and Project Financing}

Facility heads obtained skills in providing leadership to site HIV programs, meeting targets, submitting quality reports and provided supportive supervision to staff for HIV service delivery and facilities received monthly stipends to cater for transport, communication and basic consumables. Funders' requirements were upheld including finance books documentation, adherence to submission timelines and principles of allowable expenses.

\subsubsection{Data Collection and Monitoring/Evaluation Systems}

The sites records staff consisting clerks, CHWs, PLHIVs enrolled clients into HCT, PMTCT and ART, received support till proficiency in allotting unique identifiers, folders stored in cabinets and off-site data backup maintained. National M/E tools and patient folders were received and populated with HCT, PMTCT and ART at each site with support from MUHIN M/E focal person. National MNCH registers were provided for each facility with improved documentation of maternity records.

\subsection{HIV Clinical Service Delivery}

The optimization of facilities, cleanliness and infection control measures were established and use of sharps disposal, coloured bin bags, proper disposal of laboratory and delivery waste was upheld at sites. They used appropriate instruments at delivery, used EmONC drugs and procedures and referred to Job aids, charts, standard operating procedures and National guidelines provided for them. These spoke to improved $\mathrm{MNCH}$ service delivery. Drug and Commodity logistic systems and regular supplies commenced, using the hub and spoke model. Receive of rapid test kits, ARVs and drugs for Opportunistic infections like Cotrimoxazole and distribution commenced with JUTH and Lead IP program pharmacists supporting MUHIN to generate monthly forecast and distribute drugs to its sites.

The HIV services were commenced and integrated into ANC, labour ward, postnatal and child health service points and included HCT and ARV to mother baby pairs for PMTCT for those testing positive at each facility. Provider initiated counseling and testing was provided to also men, non-pregnant women and children at the immunization clinics, OPDs and community outreaches. Three Point of care (POC) equipment would be provided for the three ART sites, and sample logging commenced at OLA hospital, a comprehensive ART site, for viral load and DNA PCR (Early Infant diagnosis). Samples were pooled from PMTCT sites to ART sites, then transported by MUHIN laboratory focal person, with subsequent retrieval of results and distribution to sites through the hubs.

\subsubsection{HCT and Prevention}

Number of individuals counselled, tested and received results in MUHIN facilities were 7193 adults and 23 children, and 253 adults and 2 children were HIV positive. However 137 adults were known PLHIVs who wanted to be retested or where and experiencing challenges with assessing treatment. MUHIN provided three HCT outreaches in Angwan-Rogo and Gangare communities in collaboration with FOMWAN, who created awareness and tracking. They provided HCT and results to 803 clients ( 278 males and 525 females), and five clients tested positive ( 3 males and 2 females) and were referred for evaluation and treatment MUHIN ART site. HIV prevention messages were provided to all clients.

Post Exposure Prophylaxis (PEP) was provided to 2 MUHIN facility staff who had occupational exposure to HIV. They completed treatment and were HIV negative after retesting three months after.

\subsubsection{PMTCT}

A total of 3,293 women were assessing ANC at the 28 MUHIN facilities and were all offered HCT and same day results, with option to decline. The number of pregnant women who accepted the test and thereafter had known HIV status were 3,094 (acceptance rate of 93.9\%), and 34 tested positive and were counselled for PMTCT enrolment. However 16 of them previously knew their status and had commenced ARVs but were not regular on follow up because of the crises. They requested transfer to MUHIN sites for security and proximity and received ARVS and PMTCT/MNCH services and transfer processes were commenced. Seventeen ARV naïve women were enrolled into PMTCT and provided triple ARVs according to Option B of National HIV Guidelines, 14 whose $\mathrm{CD}_{4}>350$ cell $/ \mathrm{mm} 3$ for PMTCT purposes and 3 with $\mathrm{CD}_{4}<350$ cell $/ \mathrm{mm} 3$ for their disease. The only woman yet to enrol was being tracked by CORPs. Couple HCT and results were provided for 38 couples who tested negative, and all women received contraception counselling. One woman tested positive immediately after delivery and was commenced on ARVs. A total of 5 HIV exposed baby received Nevirapine Suspension for 6 weeks and cotrimoxazole prophylaxis thereafter. Samples of 4 HIV exposed infants sent for EID by DNA PCR and all returned negative. CORPs are tracking one baby.

\subsubsection{Care and Support}

Number of HIV infected persons enrolled into clinical care were 69 adults and 2 children. Condoms were distributed to 45 PLHIVs in each of July and August, while Long Lasting Insecticide Treated Nets, Buckets and zero-B water purifier for fifty PLHIVs and their families. Adherence counselling is provided at every visit by the dispensing health worker and 
adherence staff or expert patient (PLHIV). Home visits were made to 21 PLHIVs, while phones calls to provide support and drug adherence to 47 patients. MUHIN support group was started in June 2014 and held 3 monthly meetings, had 22 members and ongoing advocacy.

\subsubsection{Adult Treatment}

Number of HIV infected patients who had $\mathrm{CD}_{4}$ $<350$ cells $/ \mathrm{mm}^{3}$ commenced on ART were 43 (40 nonpregnant adults and 3 pregnant women), and they received cotrimoxazole prophylaxis. Weekly supervisory visit to all MUHIN ART/PMTCT sites was provided by MUHIN Clinical officer.

\subsubsection{Paediatric Treatment}

Two children of HIV positive mothers tested HIV positive and were commenced on cotrimoxazole prophylaxis, had $\mathrm{CD}_{4} \%$ tests and commenced on ARVs according to National Paediatric HIV Guidelines. They received follow-up at same MUHIN facilities with their mothers.

\subsubsection{Tuberculosis HIV Co-infection}

All patients were screened for Pulmonary Tuberculosis (PTB) according to National guidelines, with symptoms, sputum Acid fact Bacilli (AFB) and/or chest X-ray as indicated. A total of Forty nine chest X-rays and seven sputum AFB screenings were performed according to guidelines. MUHIN identified 6 cases of TB/HIV coinfection cases who were referred and commenced anti TB drugs at different DOTS sites, with two mortalities from severe disease died and late diagnosis. Efforts to secure a MUHIN site as a DOTS centre were commenced.

\subsubsection{Laboratory Services}

Investigations for all PLHIVs on ARVs (17 Pregnant women, 40 Adults, 2 children) were pooled and transported to OLA Hospital Laboratory for Chemistries, Haematology, Viral load and $\mathrm{CD}_{4}$ count at enrolment, three months and six months visits.

\subsubsection{Strategic Information}

All sites captured MNCH and HIV indicators in national M\&E tools and MUHIN team M\&E focal person visits all sites for support. Monthly report submissions are made to the Lead IP before $10^{\text {th }}$ day of each month. The MUHIN M/E officers were integrated into the state monthly $\mathrm{M} / \mathrm{E}$ meeting and MUHIN sites were captured by LACA (Local Government AIDS Control Agency). Quality improvement and Quality Assurance (QI/QA) site visits was provided by MUHIN Clinical officers with support from the Lead IP.

\subsection{Community Oriented Resource Persons Recruitment Outcomes}

A total of fifty-two community resource persons were recruited to play complementary roles in HIV/PMTCT care continuum at MUHIN sites. The three ART sites had four women attached to each, PMTCT had three each, while sixteen HCT facilities had one each. The CORPS consisted three categories of women. The first were female community expert patients (PLHIVs) who had completed the PMTCT cascade and produced HIV negative infants. They provided demand creation, treatment support, drug adherence, linkages to care, mother-to-mother (M2M) PMTCT mentors, home based care, infant follow-up and retention support. The community TBAs created HIV awareness to their clients, they referred and escorted pregnant women for HCT/PMTCT/MNCH services at target facilities. The FOMWAN created HIV/PMTCT awareness, debunked community myths about HIV/PMTCT and paid advocacy and awareness creation visit to the Nigerian Union of Road Transport workers (NURTW). FOMWAN advocated support for wives to access PMTCT/MNCH services, assistance in transporting women in labour to health facilities, they provided referrals ANC/PMTCT referrals and gave health talks in religious gatherings.

\section{Discussion}

While biomedical innovations have made PMTCT possible, barriers to access especially in low-resourced settings remain a challenge, and in many regions with highest HIV burden and greatest number of new paediatric cases, the uptake of facility-based care by pregnant women remains low, and the continuum of care necessarily relies on the community. Community resource persons provide critical links between communities and health facilities, and provide assistance to inadequate and overstretched health workers in HIV care and follow-up. This is critical in Nigeria where the HRH crises is one of the highest worldwide, which in the face of conflict constitutes a double jeopardy. We need review capturing effective, promising practices that are community-based and/or employ community-oriented groups to improve outcomes for the prevention of vertical transmission [13].

The WHO describes task shifting as involving "the rational redistribution of tasks among health workforce teams. Specific tasks are moved, where appropriate, from highly qualified health workers to health workers with shorter training and fewer qualifications in order to make more efficient use of the available human resources for health" [14] as a means of rapidly expanding access and improving health care. The goal is "to get the right workers with the right skills in the right places doing the right things"[15] without necessarily abandoning other methods of increasing numbers of qualified health workers.

The goal of the TSTS policy is to provide universal health coverage and meet Nigeria's health needs by mobilizing available human resources to ensure equity, accessibility, and effective delivery of essential healthcare services. Recommendations however stipulate that supportive supervision and clinical mentoring will be regularly provided to all health workers within the structure and functions of health teams by staff that are competent and have supervisory skills.

In Malawi, Uganda, Lesotho, Swaziland, South Africa, Zimbabwe and others, care and treatment package for 
PLHIVs were designed for delivery by non-specialist doctors, clinical officers or nurses. This has allowed rapid scale-up and complete coverage of PMTCT services, including ART initiation, refills, pregnant women monitoring, and community treatment of malaria. Complex cases are referred to higher level care, but majority of women/infants receive comprehensive care at the primary care level. Professionals are supported by CHWs, PLHIVs and other categories of volunteers such as Community Owned Resource Persons (CORPs)/Village Health Workers who have been trained as expert clients and mentor mothers [16]. The National Guidelines for the Integration of Reproductive Health and HIV Programs (FMOH, 2008), recommend that CHWs can be trained to provide certain services at PHCs, and CORPs/village Health Workers can be trained to deliver services at the community level, including advocacy, mobilization and awareness creation, health education, HIV/AIDS, HCT, universal precautions for infection control and referral of HIV-positive clients to secondary facilities [17].

The PMTCT services were introduced in Murambinda Mission Hospital, Buhera in rural Zimbabwe. Strategies included TSTS and HCT acceptability among pregnant women remained $>90 \%$ throughout, as was observed on the MUHIN program. Collection of test results and mother-child follow-up were the most challenging activities of the programme, for which they implemented and recommend district approach and community participation as critical to develop PMTCT programmes in rural settings, even with reasonably good $\mathrm{MCH}$ service [18]. This advocates for involvement of CORPs in community PMTCT programs.

A retrospective study assessed PMTCT in sixteen pilot antenatal clinics in Jamaica by examining patient records for HIV-positive pregnant women and their babies from January to December 2001. One hundred and twenty-three of 8116 pregnant women newly tested positive during the period, with $62(1.4 \%)$ and $61(1.6 \%)$ tested positive in 2001 and 2002 respectively. One hundred and ten (77\%) and $113(83 \%)$ mothers and infants, respectively, received ARV therapy [19]. The program revealed that there were women testing when they already knew their HIV status and the rates were low among the populations, same as occurred in the MUHIN project were women who knew their status were retesting. The program revealed that completing the PMTCT cascade is still challenging. This calls for identification of high risk populations, targeted HIV testing and more vigorous tracking in HIV/PMTCT clinics.

The MUHIN project was implemented by forming relevant partnerships with relevant stakeholders. The PEARL study conducted in Cameroon, Cote d'Ivoire, South Africa, and Zambia from 2007-2009 built partnerships of PLHIVs, Village Health Teams (VHTs), government health units, and other organizations to provide awareness campaigns and HCT to couples, identifying positives and giving referrals for EID. They improved child survival through increased uptake of PMTCT services, and provided care/support to PLHIVs and their families using community based and family centered strategies [20].

The ICOBI Community PMTCT was a CDC funded five (5) year project, that covered ten Ugandan districts with estimated 2,776,188 beneficiaries and expanded uptake of PMTCT interventions by using community based strategies. The project contributed towards improved child survival by increasing PMTCT uptake and providing care/support to infected parents and children using home based community strategies [21]. The ICOBI project proposes that to successfully prevent vertical HIV transmission, countries must intervene at the community level with PMTCT interventions strategies like male involvement, peer mentorship, use of community health workers, mobile phonebased reminders, integration of PMTCT services and enhanced referrals. These are approaches similar to those utilized on MUHIN project.

\section{Conclusion}

Addressing HIV/PMTCT gaps will require detailed diagnostics that utilize all appropriate and relevant lenses to analyze the barriers in coverage, access and uptake of PMTCT services. Complexity exists when conflict and its multiple implications exist in communities where persons need to access PMTCT services. Insecurity related challenges would reinforce and worsen whatever challenges exist on ground, including inadequate $\mathrm{HRH}$, inadequate health facilities, out-of-pocket cost of healthcare and other barriers to access and uptake of PMTCT services, which occur majorly at the community level and require innovation and community based approaches. The engagement of community based resources, facilities, strategies and stakeholders enabled access to communities that were hitherto sequestrated by crises. Task shifting and sharing was used to efficiently bridge the HRH gaps.

This 6 month pilot revealed this strategy is useful, although total numbers were still considering the assessment and planning and health systems strengthening aspect of the project took almost 4 months. With funding, a lot of unmet PMTCT needs peculiar to these peculiar populations can be addressed with this platform and strategies.

\section{Lessons Learnt}

1) Barriers to access to HIV/PMTCT services can be more complex in communities affected by crises even when they are within towns.

2) Acceptability, accessibility and uptake of PMTCT services can be improved by religion and ethnicity aware strategies.

3) Task shifting and sharing is critical in providing PMTCT within communities that have restricted HCW, and PLHIVs and TBAs can play important roles.

4) Scale up of PMTCT services efforts can positively impact $\mathrm{MNCH}$ service delivery and quality. 
5) Linkage to care and retention in care is still a challenge among newly diagnosed PLHIVs requiring continual efforts.

6) Conducting integrated community outreaches is also an entry point for accessing PMTCT services, but testing needs to target most-at-risk persons/communities.

7) Strengthened teamwork and relationship between health workers and community structures and resource persons increase acceptability and utilisation of services.

8) Involving community gatekeepers, religious leaders, health service providers and community members at all levels of program implementation promotes ownership and sustainability of HIV/PMTCT services.

9) Community PLHIV support group reduces stigma and discrimination in communities and provide homebased care, adherence support, PMTCT support, awareness creation, generating demand, behavioural change communication among others.

10)Collaboration between health workers and community structures like TBAs and women groups like FOMWAN increase acceptability of PMTCT services.

11)HIV implementing partners need to consider community oriented strategies in closing PMTCT gaps in communities.

12)Stakeholders meetings and advocacy meetings with the district leadership, religious leaders and local LCs have led to increased support to the health services and more so the community structures in creating demand

\section{References}

[1] UNAIDS (2013). "90-90-90 An ambitious treatment target to help end the AIDS Epidemic," available at http://www.unaids.org/en/sites/default/files/media_asset/9090-90_en0.pdf. accessed on 11/04/2021.

[2] UNAIDS (2013). Progress Report on the global plan towards the elimination of new HIV infections among children by 2015 and keeping their mothers alive. 2013. Available at: http://www.unaids.org/en/media/unaids/contentassets/docume nts/unaidspublication/2013/20130625_progress_global_plan_ en.pdf accessed on 3/04/2021.

[3] UNAIDS (2010). HIV and conflict: Connections and the need for universal access. A Forced Migration Review special supplement. Available

https://www.unaids.org/en/resources/presscentre/featurestories /2010/october/20101027fssecurityandconflict Accessed $22 / 02 / 2021$.

[4] Plateau State at a Glance. Available at https://www.plateaustate.gov.ng/plateau/at-a-glance accessed on $23 / 02 / 2021$.

[5] National Population Commission of Nigeria (2020) https://www.citypopulation.de/php/nigeriaadmin.php?adm1id=NGA032.

[6] UNICEF (2015). Interagency Task Team HIV in Humanitarian Emergencies PMTCT in Humanitarian Settings https://www.childrenandaids.org/sites/default/files/201704/IATT_Part-2-PMTCT-in-HumanitarianSettings_2015_0.pdf.

[7] WHO (2010). Global Atlas of the Health Workforce, August 2010 .

[8] Federal Ministry of Health, Nigeria (2012). Nigeria Health Workforce Profile as of December 2012. Available at https:/Nigeriandocuments.blogspot.com assessed on 20/04/2021.

[9] Federal Ministry of Health, Nigeria (2014). Task-Shifting and Task-sharing Policy for Essential Health Care Services in Nigeria. Available at https://www.health.gov.ng/doc/TSTS.pdf assessed on $01 / 03 / 2021$.

[10] Federal Ministry of Health (2009). The National Strategic Health Development Plan Framework (2009-2015). Available at https://www.uhc2030.org assessed 17/04/2021.

[11] Jos, Nigeria Metro Area Population 1950-2021. Available at https://www.macrotrends.net>cities Assessed 19/03/2021.

[12] Federal Ministry of Health, Nigeria (2010). National Guidelines for Prevention of mother-to-child transmission (PMTCT) of HIV 2010.

[13] Marcos Y, Phelps BR, Bachman G (2012). Community strategies that improve care and retention along the prevention of mother-to-child transmission of HIV cascade: a review. J Int AIDS Soc. 2012 Jul 11; 15 Suppl 2 (Suppl 2): 17394. doi: 10.7448/IAS.15.4.17394. PMID: 22789647; PMCID: PMC3499877. Available at https://pubmed.ncbi.nlm.nih.gov/22789647/Assessed $19 / 03 / 2021$

[14] World Health Organization (2008): Task Shifting-Global Recommendations and Guidelines. 2008. Available at: http://www.who.int/healthsystems/TTR-TaskShifting.pdf Assessed on 22/01/2021.

[15] World Health Organization (2008): Working together for health: the World Health Report. 2006. Geneva, Switzerland: World Health Organization, 2006. Available at: http://www.who.int/whr/2006/whr06_en.pdf. Assessed on $22 / 01 / 2021$.

[16] Samb B, Celletti F, Holloway J, Van Damme W, Lawson L, De Cock K, Dybul M. (2007). Task shifting: An emergency response to the health workforce crisis in the era of HIV. Lessons from the past, current practice and thinking. N Engl Med 357; 24.

[17] Federal Ministry of Health (2008). National Guidelines for the integration of Reproductive health and HIV programs in Nigeria 2008.

[18] Perez, F, Mukotekwa, T, Miller, A, Orne-Gliemann, J, Glenshaw, M, Chitsike, I and Dabis, F. (2004) Implementing a rural programme of prevention of mother-to-child transmission of HIV in Zimbabwe: first 18 months of experience. PMID: 15228487. DOI: 10.1111/j.13653156.2004.01264.x Assessed on 20 /01/2021.

[19] Harvey, K. M, Figueroa, J. P, Tomlinson, J, Gebre, Y, Forbes, S, Toyloy, T, Thompson, T, Thompson, K. An assessment of mother-to-child HIV transmission prevention in 16 pilot antenatal clinics in Jamaica. PMID: 15675493. 
[20] Center for Health Market Innovations. Community Prevention of Mother to Child HIV transmission Project. Available at https://healthmarketinnovations.org/program/community-

prevention-mother-child-hiv-transmission-project Accessed $16 / 02 / 2021$.
[21] Measure Evaluation. Community Based Indicators for HIV Programs. Prevention of Mother to Child Transmission of HIV. Available

https://www.measureevaluation.org/community-basedindicators/PMTCT/pmtct assessed 22/02/2021. 